\title{
A Pulmonary Infarction Mimicking Metastatic Lung Tumor
}

\author{
Koji Teramoto, You Kawaguchi, Tetsuo Hori, Yasuhiko Ohshio, Masayuki Hashimoto, \\ Shoji Kitamura, Jun Hanaoka, Noriaki Tezuka \\ Department of Surgery, Shiga University of Medical Science, Otsu, Japan \\ E-mail:teramoto@belle.shiga-med.ac.jp \\ Received May 9, 2011; revised July 1, 2011; accepted July 22, 2011
}

\begin{abstract}
Pulmonary infarction may present as a solitary pulmonary nodule resembling a malignant pulmonary tumor. A 71-year-old woman who had undergone the surgery for abdominal malignant fibrous histiocytoma (MFH) with inflammatory nature presented with a small nodule in the right lung in computed tomographic images. Two months later, the nodule became enlarged and had an accompanying infiltrative shadow. We suspected a metastatic tumor from MFH and performed video-assisted thoracic surgery. However, subsequent pathological diagnosis of the nodule was pulmonary infarction due to thromboembolism. When a peripheral nodule is detected, pulmonary infarction should be considered as a possible diagnosis.
\end{abstract}

Keywords: Pulmonary Infarction, Lung Cancer, VATS

\section{Introduction}

Pulmonary infarction may present as a solitary pulmonary nodule, presenting radiologically as peripheral wedge-shaped parenchymal opacities [1-3]. However, in some cases, it may present as a nodule resembling a primary or metastatic lung tumor [4]. In previous reports on a pulmonary infarction, there are few reports describing its radiological findings altered with time. Here, we report a case of pulmonary nodule mimicking a metastatic tumor that was subsequently diagnosed as pulmonary infarction following video-assisted thoracic surgery (VATS). The altered radiological findings of the pulmonary nodule in our case made us suggest a metastatic tumor.

\section{Case Report}

A 71-year-old woman who had undergone surgery for abdominal malignant fibrous histiocytoma (MFH) presented with a pulmonary nodule in the right lower lobe, S8, on chest computed tomography (CT) during following-up. The nodule was $8 \times 7 \mathrm{~mm}$ in size with regular margin (Figure 1(a)). Two months later, the nodule had enlarged to $20 \times 10 \mathrm{~mm}$ in size and was accompanied by a surrounding infiltrative shadow (Figure 1(b)). Considering her past history of MFH, which had a potent in-

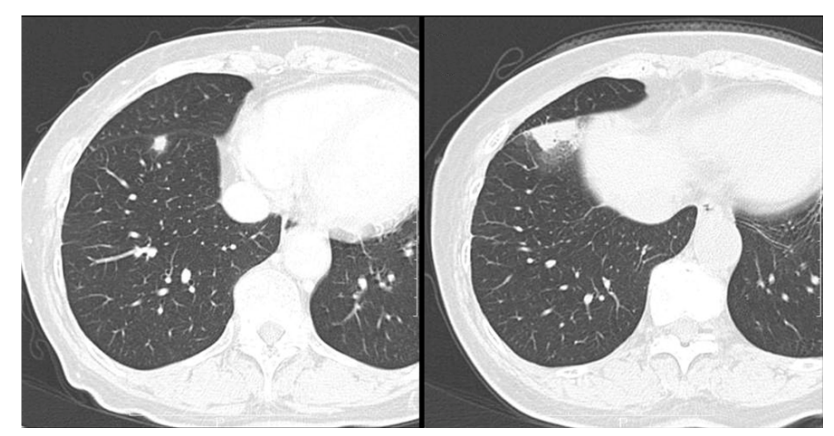

(a)

(b)

Figure 1. (a) Chest CT showing a small nodule in right lung, S8; (b) two months later, it had enlarged to be accompanied by a surrounding infiltrative shadow.

flammatory background, the nodule was suspected to be a metastatic tumor from MFH. She was subsequently referred to our department for diagnosis confirmation and removal of the nodule. Suspecting the nodule to be a metastatic tumor as well, we conducted VATS to excise it. During surgery, the visceral epipleura of the nodule was found to be thickened and grey in color. There were no findings related to the local inflammation of the nodule, that is, accumulation of reactive pleural effusion, adhesion of the nodule to the chest wall, and angiogenesis around the nodule. We could resect the nodule easily. Upon removal, the nodule was found to be of elastic hard 
consistency and $20 \times 20 \times 15 \mathrm{~mm}$ in size. The cut surface of the nodule was dark red in color (Figure 2(a)) and it was pathologically diagnosed as a pulmonary infarction caused by thromboembolism (Figure 2(b)). Malignant cells were not detected in the resected specimen.

\section{Discussion}

Pulmonary infarctions typically result from pulmonary thromboembolism. Other than this, non-thromboembolic causes of pulmonary infarctions include pulmonary infections, diffuse alveolar damage, pulmonary torsion, lung cancer, amyloidosis, bronchial artery embolization therapy, and intravenous catheter embolization [5]. In this case, pulmonary infarctions resulted from pulmonary thromboembolism but causes of thrombus were not fully revealed. The patient had lower risk to occur the thrombus, that is, she had neither varix of the lower limb nor atrial fibrillation. And she took an oral anti-coaglant drug

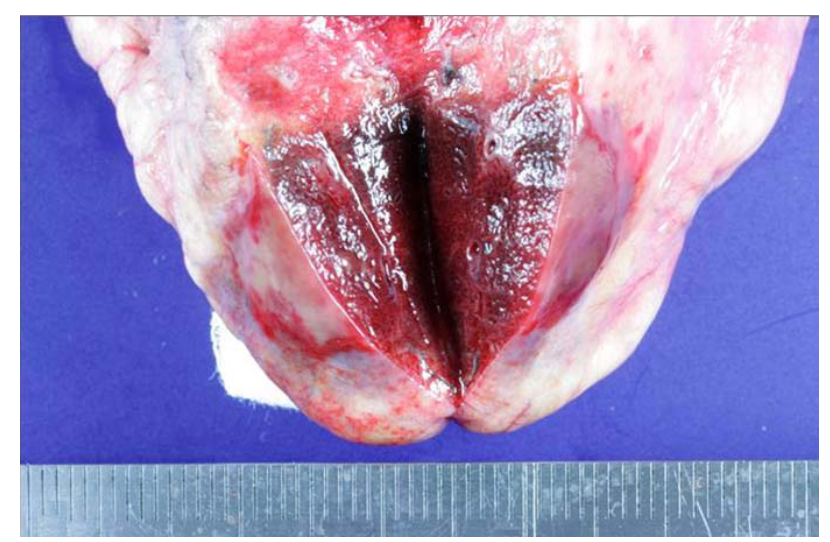

(a)

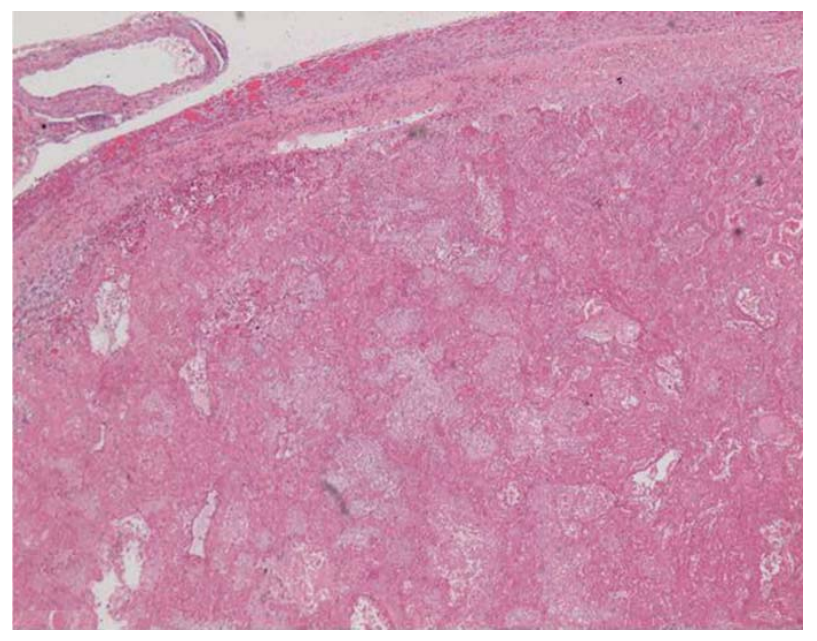

(b)

Figure 2. (a) The resected specimen of the pulmonary nodule; (b) HE-staining of the nodule demonstrating necrotic tissue at the subpleural region. which was for prevention from cerebral infarction. A speculation about the cause of thrombus is that past surgery for MFH might set off occurrence of deep vein thrombus.

Radiologically, pulmonary infarction appears as hump-shaped opacities located in the subpleural space [1-3]. Pulmonary infarctions are generally polyhedral, conform to a secondary pulmonary lobule, and have a bronchovascular connection directed toward the hilum [1]. However, they may assume various other shapes, partly due to associated hemorrhage and edema. In this case, a solitary small nodule was initially detected on chest CT. Two months later, the nodule had become enlarged and had an accompanying infiltrative shadow. Taking into consideration that the patient's MFH had inflammatory in nature, we suspected that the nodule might to be a metastatic tumor from MFH. As a result of the confusing change in findings on chest $\mathrm{CT}$ over a period of two months, we did not conceive of pulmonary infarction as a differential diagnosis. We assumed that the small nodule initially detected had induced inflammation or congestion in the area resulting in the infiltrative shadow detected.

In a review of the previous cases, George and colleagues reported 43 instances of pulmonary infarction [4], and six of which had features suggestive of lung cancer. Three of six cases were enhanced positively on contrast-enhanced CT, two cases were hyper-metabolic on positron emission tomography (PET). More information may be given by not contrast-enhanced but multi-sliced CT for preoperative diagnosis of pulmonary infarction. PET seems to contribute little to diagnosis because of accompanied inflammation of pulmonary infarction. Takahashi and colleagues reported that pulmonary infarction should be considered as a differential diagnosis when peripheral pulmonary nodules or masses are located in the same lobes as the primary cancer [6]. Therefore, we maintain that biopsy by minimally invasive VATS is the most reliable method to diagnose pulmonary infarction.

\section{Conclusions}

Based on this report, we suggest that in the case of pulmonary nodules located peripherally in subpleural regions, pulmonary infarction should be considered as a differential diagnosis in patients with a past history of thromboembolism.

\section{References}

[1] J. Balakrishnan, M. A. Meziane, S. S. Siegelman and E. K. Fishman, "Pulmonary Infarction: CT Appearance with Pathologic Correlation,” Journal of Computer Assisted 
Tomography, Vol. 13, No. 6, 1989, pp. 941-945. doi:10.1097/00004728-198911000-00001

[2] S. M. Greaves, E. M. Hart and D. R. Aberle, "CT of Pulmonary Thromboembolism," Seminars in Ultrasound, CT and MRI, Vol. 18, No. 5, 1997, pp. 323-337. doi:10.1016/S0887-2171(97)90010-X

[3] P.T. Johnson, R. J. Wechsler, A. M. Salazar, A. M. Fisher, L. N. Nazarian and R. M. Steiner, "Spiral CT of Acute Pulmonary Thromboembolism: Evaluation of Pleuroparenchymalabnormalities," Journal of Computer Assisted Tomography, Vol. 23, No. 3, 1999, pp. 369-373. doi:10.1097/00004728-199905000-00009

[4] C. J. George, H. D. Tazelaar, S. J. Swensen and J. H. Ryu,
“Clinicoradiological Features of Pulmonary Infarctions Mimicking Lung Cancer,” Mayo Clinic Proceedings, Vol. 79, No. 7, 2004, pp. 895-898. doi:10.4065/79.7.895

[5] J. G. Parambil, C. D. Savci, H. D. Tazelaar and J. H. Ryu, "Causes and Presenting Features of Pulmonary Infarctions in 43 Cases Identified by Surgical Lung Biopsy," Chest, Vol. 127, No. 4, 2005, pp. 1178-1183. doi:10.1378/chest.127.4.1178

[6] M. Takahashi, Y. Murakami, N. Nitta, K. Murata, N. Tezuka, S. Fujino and H. Okabe, "Pulmonary Infarction Associated with Bronchogenic Carcinoma,” Radiation Medicine, Vol. 26, No. 2, 2008, pp. 76-80.

doi:10.1007/s11604-007-0192-9 